\title{
A Neural Model of Demyelination of the Mouse Spinal Cord
}

\author{
Biljana Petreska and Yossi Yovel
}

\begin{abstract}
This paper presents a neural network model of demyelination of the mouse motor pathways, coupled to a central pattern generation (CPG) model for quadruped walking. Demyelination is the degradation of the myelin layer covering the axons which can be caused by several neurodegenerative autoimmune diseases such as multiple sclerosis. We use this model - to our knowledge first of its kind - to investigate the locomotion deficits that appear following demyelination of axons in the spinal cord. Our model meets several physiological and behavioral results and predicts that whereas locomotion can still occur at high percentages of demyelination damage, the distribution and location of the lesion are the most critical factors for the locomotor performance.
\end{abstract}

\section{INTRODUCTION}

Artificial neural networks are a well established tool commonly used in research that aims to gain a better understanding of biological neural networks. Their basic elements include simple processing elements (simulating the neurons) connected in a network (representing the axons). The neurons act upon each other according to a set of simple rules, however, the entire system can exhibit complex global behavior. Although stemming from a biological background, neural network models have never taken into account the role played by the insulating layer of the axons, known as myelin, thus always assuming perfect insulation.

Myelin is an electrically insulating phospholipid layer that surrounds the axons of most neurons in the body. Myelin is created by different cells in the central and the peripheral nervous system, but it serves a similar task in both cases. Its main functions are to increase the resistance of the cell membrane and to decrease its capacitance and thus to enhance the propagation speed of the electric signal along the axon, and to prevent it from leaking out. A degradation of the myelin layer, termed demyelination, can be caused by several neurodegenerative autoimmune diseases such as multiple sclerosis (MS) as well as by a postponing source like heavy metals [1]. On the neurophysiological level a degradation of the myelin layer slows down the signal's propagation speed, and decrease its amplitude. In addition, imperfect insulation of two adjacent axons can lead to a transfer of electric potential from one to the other, also known

Biljana Petreska is with the Learning Algorithms and Systems Laboratory, Ecole Polytechnique Fédérale de Lausanne (EPFL), Switzerland. Yossi Yovel is with the Department of Animal Physiology, Tübingen University, Germany (email: biljana.petreska@a3.epfl.ch, yossiyovel@hotmail.com).

This work was supported by the Santa Fe Institute through NSF Grant No. 0200500 entitled "A Broad Research Program in the Sciences of Complexity", the Human Resources and Mobility Activity Marie Curie Host Fellowships for Early Stage Research Training under contract MEST-CT2004-504321 'PERACT' by the European Union and by the Sport and Rehabilitation Engineering Program at EPFL.

Both authors contributed equally to this work and were ordered alphabetically. as the crosstalk phenomenon. The physiological symptoms of demyelination depend on the region of the damage, and can include impaired vision or motion. In this paper, for the first time to our knowledge, we try to introduce a framework for demyelination into an artificial neural network model. To test it we apply it to a network simulating locomotion.

It is now well known that locomotion in vertebrates is generated by central pattern generators (CPGs) in the spinal cord [2]. The CPG is a dynamical neural unit that receives tonic input drive from the brainstem and generates the oscillatory patterns needed for walking, swimming or other gaits, according to this input [3], [5], [4], [6]. In this paper we simulate the activity of motoneurons (i.e., neurons that activate the muscles) in the spinal cord of the mouse using a potential, although simplified, CPG model based on the Ellias-Grossberg oscillator (described in more detail in section II) [7].

\section{MODEL DESCRIPTION}

We developed a neural model of the last part of the mouse's ventral corticospinal tract, namely of the axons starting at the brainstem control neurons and connecting to the motoneurons in the CPGs of the limbs (shown on Fig. 1.A). We then impair the signal conducted through these axons in a way that is similar to the result of demyelination and observe how this affects locomotion.

\section{A. Model for quadruped walking}

The CPG model used in this work is a version of the ElliasGrossberg oscillator whose neurons obey the biologically inspired Hodgkin-Huxley equations [7]. The key feature of this model is different time scales of operation between fast excitatory on-center and slow inhibitory off-surround neurons. The motoneurons of a CPG unit related to one limb receive a single tonic input that controls the frequency and phase relationships with the CPG units of the other limbs, so that gait transitions can be triggered at particular activation levels. Importantly, as the input signal to the motoneurons is increased, the system produces the same gait with increased speed and at a particular level switches to a different walking gait - walk, trot, pace and gallop - in the same order as they appear in the cat. Here we only use the walking pattern of the model to simulate the behavior of a mouse on a rod rotating at a constant speed, as we assume that walking is the most adapted gait considering the size and speed of the rotarod. The model for quadruped walking comprises four oscillators, each related to one limb (see Fig. 1.A). Each oscillator $i$ (1 indexes the left fore limb oscillator, 2 the right fore limb, 3 the left hind limb and finally 4 refers to the right hind limb oscillator) is composed of one excitatory motoneuron $x_{i}$ and 
one inhibitory motoneuron $y_{i}$ whose membrane potentials are governed by the following equations:

$$
\begin{gathered}
\left\{\begin{array}{l}
\dot{x}_{i}=-\alpha x_{i}-\left(\beta+x_{i}\right) \sum_{j} d_{i j} g\left(y_{j}\right)+\left(\gamma-x_{i}\right)\left[f\left(x_{i}\right)+I\right] \\
\dot{y}_{i}=\epsilon\left(\left(1-y_{i}\right)\left[x_{i}\right]^{+}-y_{i}\right)
\end{array}\right. \\
{[w]^{+}=\max (w, 0), \quad i, j \in 1, \ldots, 4 .}
\end{gathered}
$$

where $I \in R[0,0.5]$ is the tonic brainstem input. $\alpha, \beta, \gamma$, $\epsilon \in R[0,2.5]$ are scaling parameters (the values chosen are shown in Table I) and $d_{i j}$ is the strength of the connection between the inhibitory motoneuron $y_{j}$ to the excitatory motoneuron $x_{i}$. The connectivity for the 4 limb CPGs is shown in Figure 1.B and the values chosen for the entries of this connectivity matrix are given in Table II. The output of an excitatory / inhibitory motoneuron is a rectified sigmoid function $f / g$ of their respective membrane potentials $x_{i}$ and $y_{j}$, and corresponds to the firing rate of the motoneuron:

$$
f\left(x_{i}\right)=\frac{r_{x}\left(\left[x_{i}\right]^{+}\right)^{2}}{s_{x}+\left(\left[x_{i}\right]^{+}\right)^{2}} \quad g\left(y_{j}\right)=\frac{r_{y}\left(\left[y_{j}\right]^{+}\right)^{2}}{s_{y}+\left(\left[y_{j}\right]^{+}\right)^{2}}
$$

where $f(x) \in R\left[0, r_{x}\right]$ and $g(y) \in R\left[0, r_{y}\right]$. The values of the parameters $r_{x}, r_{y}, s_{x}, s_{y} \in R[0,10]$ are given in Table I. The oscillator is assumed to govern the extension and flexion of the limb muscles $i$ through the output activity $f\left(x_{i}\right)$ of its excitatory neuron $x_{i}$.

TABLE I

PARAMETER VAlues (PLEASE REFER TO SECTION II-A FOR MORE DETAILS)

\begin{tabular}{|c|c|c|c|}
\hline$\alpha=1$ & $\beta=1.05$ & $\gamma=2.5$ & $\epsilon=1.5$ \\
\hline$r_{x}=9.8$ & $s_{x}=0.5$ & $r_{y}=3.9$ & $s_{y}=0.5$ \\
\hline$\tau_{1}=0 s$ & $\tau_{2}=0.05 s$ & $\tau_{3}=0.1 s$ & $\tau_{4}=0.15 s$ \\
\hline
\end{tabular}

TABLE II

CONNECTIVITY MATRIX BETWEen THE 4 LIMB CPGS. THE OSCILlators ARE NUMBERED AS FOLLOWS: 1 IS THE LEFT FORE LIMB, 2 THE RIGHT FORE LIMB, 3 THE LEFT HIND LIMB AND 4 IS THE RIGHT HIND LIMB OSCILLATOR

\begin{tabular}{|c|c|c|c|}
\hline$d_{11}=1$ & $d_{12}=0.3$ & $d_{13}=0$ & $d_{14}=0.3$ \\
$d_{21}=0.3$ & $d_{22}=1$ & $d_{23}=0.3$ & $d_{24}=0$ \\
$d_{31}=0.3$ & $d_{32}=0$ & $d_{33}=1$ & $d_{34}=0.3$ \\
$d_{41}=0$ & $d_{42}=0.3$ & $d_{43}=0.3$ & $d_{44}=1$ \\
\hline
\end{tabular}

Interlimb oscillations are obtained by introducing stereotyped temporal asymmetries in the arrival time of the brainstem control signal at each leg, the latencies $\tau_{i}$ from onset time are given in the third row of Table I.

We first extended this model so that the brainstem tonic control signal $I$ arrives at the level of the excitatory motoneurons in a distributed fashion, similar to what happens in the living organism (see Fig. 1.A). The input signal $I_{i}$ to the CPG's excitatory motoneuron with index $i$ is thus a weighted sum of 1000 brainstem control signals that travel through the spinal cord axons. To be more specific, 4 axon
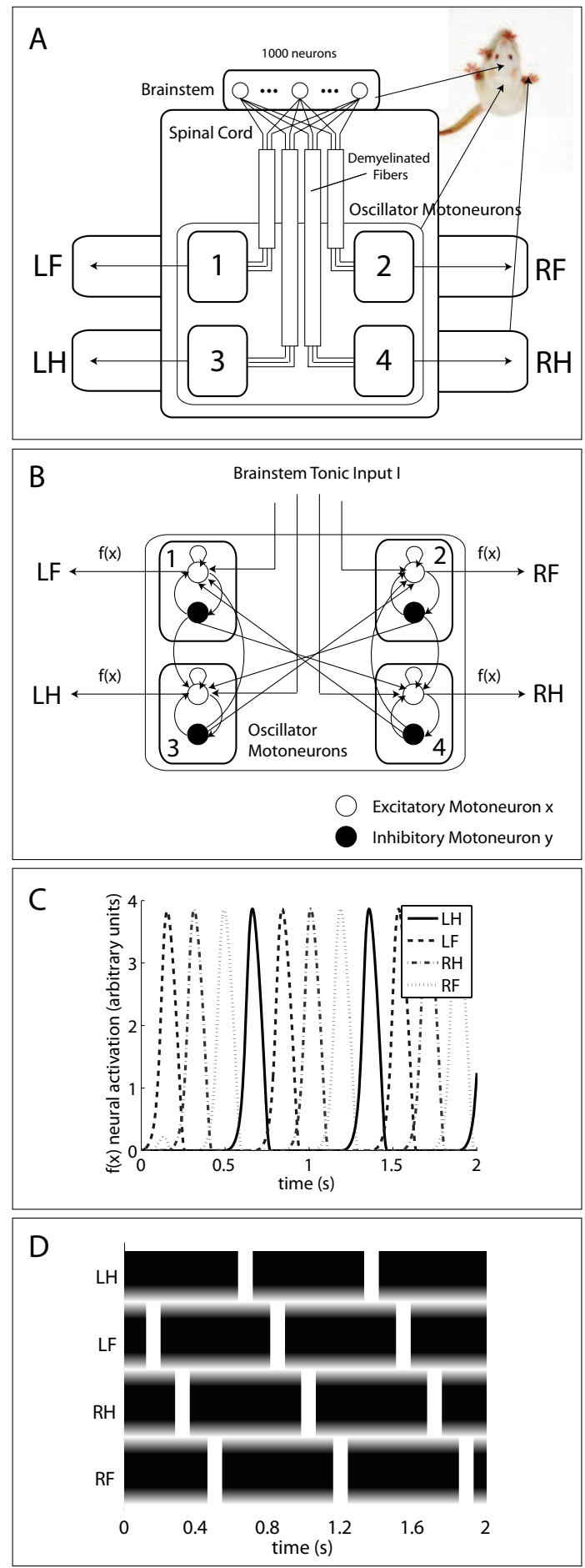

Fig. 1. A, A general schema of the model. $B$, The connectivity within and between the 4 limb CPG oscillators. $C$, The output activity of the limb oscillators for walking ( $\mathrm{L}$ is left, $\mathrm{R}$ right, $\mathrm{F}$ fore limb and $\mathrm{H}$ hind limb), the brainstem tonic input was set to 0.1 . $D$, A Pearson diagram of the simulated walking gait of the mouse, steps are represented in white and correspond to periods of time when the output activity of the excitatory motoneuron exceeded the threshold of 2 . 


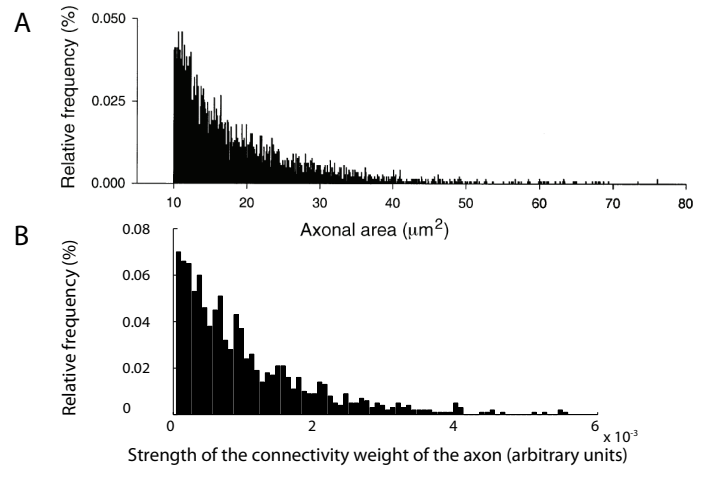

Fig. 2. A, An example of the distribution of axons of different sizes in the spinal cord, taken from [8]. $B$, In order to match the biological distribution we have distributed the connection weights between brainstem control neurons and CPG motoneurons using the exponential distribution $e^{-\rho x}$, with $\rho$ set to 0.4

bundles with different lengths and thus different conduction times, leave each of the 1000 control neurons and connect it to all 4 motor units, such that the same total brainstem control signal travels through 4 separate bundles of axons, each of which controls a specific leg:

$$
\begin{aligned}
& I_{i}(t)=\sum_{k} w_{i k} b_{k}(t) \quad i \in 1, \ldots, 4 ; \quad k \in 1,2, \ldots, 1000 \\
& \text { u.c. } \quad \sum_{k} w_{i k}=1 \quad \forall k ; \quad b_{k}=I .
\end{aligned}
$$

where $b_{k}$ is the activity of the $k^{\text {th }}$ brain stem neuron devoted to the control of limb movement and $w_{i k}$ is the strength or weight of the connection between this neuron and the excitatory motoneuron $x_{i}$ of limb CPG $i$. The strength of connectivity between two neurons can be biologically interpreted as the diameter of their connecting axon. We distributed the values of the spinal cord weights so that they would match the biological weights distribution found in [8], i.e., we used an exponential distribution to simulate numerous small axons and rare large axons as shown on Figure 2.

\section{B. Demyelination model}

Demyelination is believed to have principally two different effects: (i) leakage and crosstalk and (ii) a slow down in the signal propagation speed [1] (see also section I).

i) As a result of the insufficient insulation, the signal might decrease due to a leakage out of the axon. In the case when a second demyelinated axon is in the vicinity it might be affected by the leakage so that its potential will be changed. This means that the signals from two demyelinated neighboring axons influence each other. We assumed that this influence is additive and proportional to the amount of demyelination of the axons. Thus the potential change due to crosstalk and leakage $c_{i k}$ of an axon $w_{i k}$ is expressed as a term that sums the signal leaking from neighboring axons (depending on their respective demyelination factors) minus the total signal leaking from the axon itself, such that a fully myelinated axon will not diffuse nor absorb:

$$
\begin{aligned}
c_{i k}(t) & =m_{i k}\left(\frac{1}{|N|} \sum_{w_{j m} \in N} m_{j m} w_{j m} b_{m}(t)-w_{i k} b_{k}(t)\right) \\
i & \in 1, \ldots, 4 ; \quad k \in 1,2, \ldots, 1000 .
\end{aligned}
$$

where $m_{i k} \in R[0,1]$ corresponds to the percentage of demyelination of an axon ( 0 is for fully myelinated and 1 is for fully demyelinated), $w_{i k}$ is the strength of an axon connecting the brainstem control neuron $b_{k}$ and excitatory motoneuron $x_{i}$ and corresponds roughly to the diameter of the axon. $N$ is the set of neighboring axons of $w_{i k}$, with cardinal number $|\mathrm{N}|$, that follow the spatial model of parallel bundles of 1000 axons adopted previously (shown on Fig. 1.A). The $|N|$ neighbors of an axon $w_{i k}$ are the axons from the same bundle $i$ and from the other ipsilateral limb bundle whose indices $k \in[k-n, k+n]$, throughout the simulations $n$ was set to 100 which corresponded to $10 \%$ of the total number of axons in the bundle and for simplicity edges were treated circularly. Thus, according to our model, crosstalk can also occur between axons of different bundles if they control two ipsilateral limbs and have similar indices.

ii) Demyelination causes also slowing of the signal and thus a delay in the conduction time of the signal through the spinal cord, that is again assumed to be proportional to the amount of demyelination:

$$
\tau_{i k}=\tau * m_{i k}
$$

meaning that a $100 \%$ demyelination would result in slowing the signal for the maximal latency $\tau(1 \mathrm{~ms})$, where $\tau$ is the maximal traveling time delay caused by demyelination and was chosen to match the biological value found in [8].

After introducing the distributed input, demyelination crosstalk and delay, part 1 of equation 1 representing the membrane potential of an excitatory motoneuron becomes:

$$
\begin{aligned}
\dot{x_{i}}= & -\alpha x_{i}-\left(\beta+x_{i}\right) \sum_{j} d_{i j} g\left(y_{j}\right)+ \\
& \left(\gamma-x_{i}\right)\left(f\left(x_{i}\right)+\sum_{k} w_{i k} b_{k}\left(t-\tau_{i k}\right)+c_{i}\left(t-\tau_{i k}\right)\right) \\
& i \in 1, \ldots, 4 ; \quad k \in 1,2, \ldots, 1000 .
\end{aligned}
$$

\section{DATA ANALYSIS}

To analyze the effects of demyelination on our model we set the tonic brainstem input and CPGs connections so that the mouse would normally walk or run and measured the following quantities: relative phases between the front limbs of the mouse, amplitude of steps and motoneuron activities. In order to ease the analysis we transformed our motoneuron output signal into a binary raster plot (see Fig. 1.D). A step (white rectangle in the figure) was defined as the entire region in which the signal exceeded a certain threshold level. It is 
A
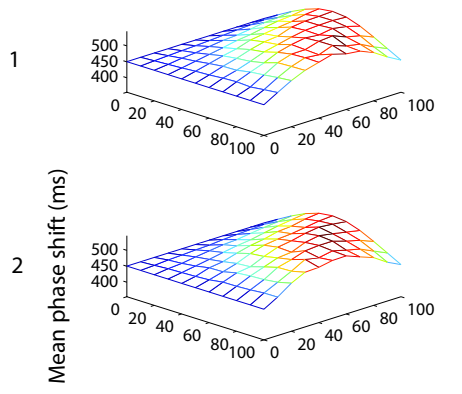

3

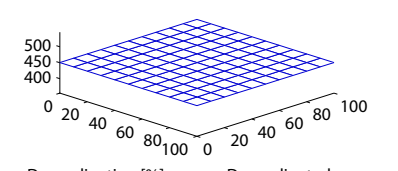

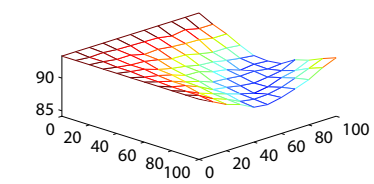
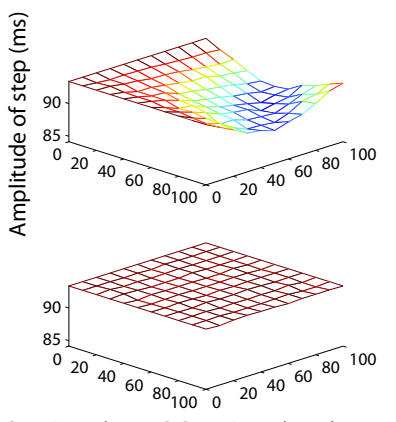

Fig. 3. A, Relative phase between the left and right front limbs and $B$, Mean amplitude of steps following a uniform lesion given for different percentages of demyelination and demyelinated neurons. The first row shows the effect of both crosstalk with leakage and time delay, the second row shows the effects of crosstalk with leakage and the third row the effects of time delay alone. The tonic brainstem input was set to 0.1 and threshold was set to 2 .

worth noticing that changing the threshold would change the sizes of the steps and consequently change the borders of what we define as walking for instance. It will not however, have any real influence on the observed phenomena. We used the raster plot to calculate the mean amplitude of steps and the shift between different legs in time units. The gait shown in the diagram in Fig. 1.D is walking, as the limbs are activated in a precise cyclic order: left hind limb - left fore limb - right hind limb - right fore limb, whereas running would correspond to an activation of the limbs in the same precise order but a higher frequency (refer to [7] for more detail). We did not consider other gaits than walking and running as we estimated them unappropriate for the execution of the rotarod task. We consider that the system is not walking anymore when it does not meet one of the following criteria: (i) the order of activation of the limbs is equivalent to the walking gait shown in Fig. 1.D (ii) the step of one limb ends before the other limbs initiate their step and (iii) the average amplitude of the steps is the same for different limbs so that the distance traveled by one leg is not too big or too small compared to that of the other limbs.

\section{REsults}

In this part we present the results obtained for uniform, focal asymmetric and biologically distributed lesions. We also describe the particular contributions of crosstalk with leakage and time delay on the behavior of the model as well as the effect of the distribution of the demyelination lesion on the locomotion patterns of the CPGs.

\section{A. Systematic investigation in the case of a uniform lesion:} a toy example

In order to gain some insight into the direct consequences of different levels of demyelination we first impaired the spinal cord weights $w_{i k}$ in a systematic and uniform manner, i.e., all affected axons had the same level of demyelination. The result is shown on Fig. 3.1 for the relative phase between the two front limbs (Fig. 3.A) and for the mean amplitude of steps (Fig. 3.B) at different percentages of demyelination. As it can be observed, until about $70 \%$ of demyelinated neurons, demyelination causes an increase in the relative phase between the two front limbs and a decrease in the amplitude of steps, thus slowing the walking gait. This effect is probably due to the leakage component of our model. When the damage is above $70 \%$ the tendency is reversed depending on the demyelination level: the relative phase decreases and amplitude of steps increases but never returns to the original values of the non-demyelinated system. This is due to the fact that with high percentages of demyelination and demyelinated neurons, the crosstalk component has more influence than the leakage. The variations in the quantities measured are gradual. The model thus predicts that the more the spinal cord of the mouse is demyelinated, the slower its walking is, such that the mouse has to increase its brainstem input signal in order to maintain a certain speed. The pattern obtained for this tonic brainstem input is always walking, whereas for higher brainstem inputs and at high demyelination percentages we also observe non-walking locomotion patterns where several limbs are simultaneously active, or walking locomotion patterns that initiate with a non-walking transient such as the one shown on Fig. 4 and explained in more detail in Section IV-B. 


\section{B. Comparison of contributions of crosstalk and time delay}

In a second step we compared the contributions of crosstalk with leakage to time delay separately in order to see how each of these terms affects the walking gate. We do this by eliminating the effect of the other factor.

The effects of crosstalk and leakage alone: Crosstalk created transient patterns, namely a few non coordinated steps before the walking stabilized as shown in Fig. 4. This is probably due to perturbations of the onset of the brainstem input signal at the level of the limbs CPGs. Indeed, in some cases, demyelinated axons that did not convey any signal at a present time (e.g., axons that would control the last leg to move) received portions of the signals of their demyelinated neighbors (crosstalk across ipsilateral bundles) such that a different gait cycle was initiated. However, the system exhibited walking behavior in the long run. Leakage decreased the total amount of input signal that arrived at the level of the motoneurons, which by construction of the walking model (see Section II-A) corresponded to slowing of the walking gait. In addition, we found that only crosstalk and leakage provoked an increase in the relative phase between the two frontal limbs cycles and a decrease in the mean amplitude of steps, whereas delay did not perturb these quantities (see Fig.3.2 and Fig.3.3).

The effect of time delay alone: With time delay alone, the walking was perfect but started later (see Fig. 3.3 and Fig. 5). It is important to note that this result is valid only for a uniform lesion, meaning that each limb is delayed by an equal amount of time, which leads to the same temporal asymmetries between the onsets of the brainstem input signals at the level of the limbs CPGs as in the normal case and thus the same walking gait is initiated.

\section{A focal asymmetric lesion}

In another experiment we demyelinated only a single axon bundle that controls a single mouse limb. The results of this action show that the location and symmetry of the lesion is a critical parameter, because the system stopped functioning already at very low demyelination percentages in the same conditions as the ones used in Section IV-A where the system always walked.

\section{A biological lesion}

Finally we tried to reproduce a typical biological lesion similar to the ones observed in [8] caused by a virus. Larger axons (i.e., with strong connectivity weights in our model) were more frequently demyelinated than small axons. The lesions were created in a focal manner, meaning that for a given number of lesions, the center of each lesion was randomly selected, and the axons surrounding it were demyelinated by a constant of $90 \%$. Beside the number of lesions we also varied the size of the lesions (same for all the lesions within one experiment) by changing the number of demyelinated neighbors. The result was very dependent on the positions of the lesions and of the distribution of the connection weights (chosen randomly) such that the system exhibited a
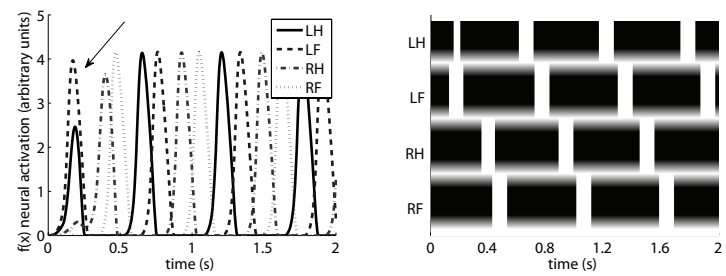

Fig. 4. In some cases, crosstalk provoked a transient non walking pattern at the initiation of the movement: demyelinated axons that did not convey any signal (e.g., axons that would control the last leg to move) received portions of the signals of their demyelinated neighbors (crosstalk across ipsilateral bundles) such that a different gait cycle was initiated. However the system usually stabilized in a walking behavior (tonic brainstem input was set to 0.3 and threshold was set to 2 ).

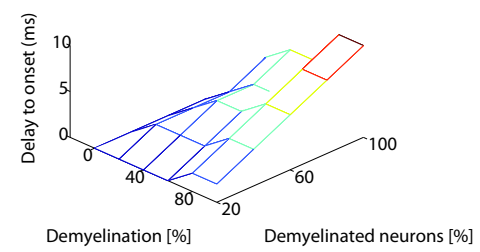

Fig. 5. Time delay alone (no crosstalk and leakage) did not affect the phase relation nor step amplitude as shown on Fig. 3.3, so that the functionality of the walking was entirely preserved, however the latency to onset time of the movement was delayed as shown here. Same conditions as in Fig. 3.

much richer repertoire of behavior than previously. We could observe many different non-walking activation patterns from which we show two examples in Fig. 7 that are obviously not representative of all the situations we have encountered. It should be noted that the effect of crosstalk was less critical in the biological lesion since the probability that two demyelinated axons are in the vicinity of each other is much smaller then in the uniform lesion.

\section{DISCUSSION}

The most remarkable result of our model is its robustness. It was very surprising to find that even with very high demyelination levels, in the case of a uniform lesion for example, the system's behavior was perturbed only transiently.
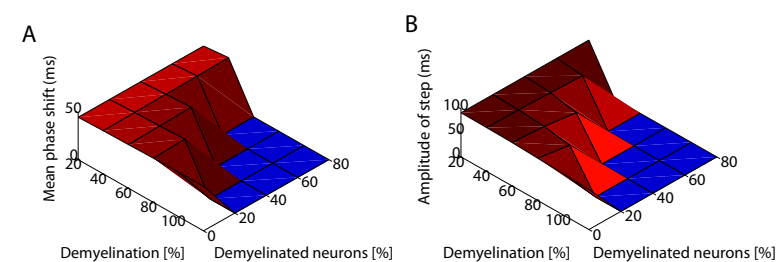

Fig. 6. Non-walking states in the focal asymmetric lesion were observed for certain demyelination percentages when the phase shift between limbs dropped to zero (A) or the step size dropped to zero (B), same conditions as in Fig. 3. 

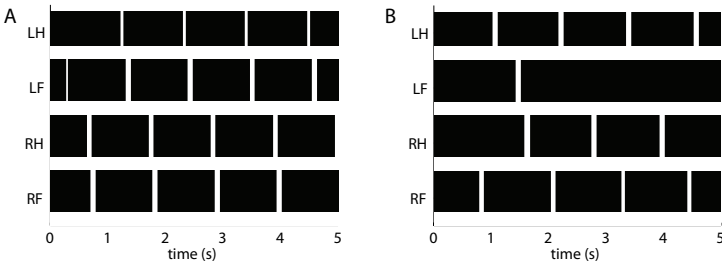

Fig. 7. Examples of locomotion patterns following biological lesions randomly distributed and affecting mostly larger axons. A, A non-walking pattern where the mouse lifts his front and hind ipsilateral limbs simultaneously (the number of lesions is 16 and number of neighbors is 150). B, A non-walking pattern where the oscillation is lost for only one limb (number of lesions is 36 and number of neighbors is 70 ).

Thus, following a short transient phase of an unidentified gait pattern the system would settle down in a walking cycle. We believe that this is an intrinsic property of the dynamical system of coupled CPGs, emphasizing a large parameter space for the walking attractor limit cycle. Our model has also met several biological and behavioral results such as delayed onset of movement and shorter intervals of walking behavior with increasing demyelination of medium-to-large axons.

In addition, we could separate the individual contributions of the two main effects following demyelination which are axonal crosstalk and delay in conduction time. We found that, in the case of a uniform lesion, delay did not perturb the amplitudes of steps nor the phase relationships between the activations of different legs, i.e., the walking cycle was the same except for the later initiation. Depending on the demyelination percentage, this would result in a mouse that may manage to initiate the walking cycle on time and not show any other deficit such as falling from a rotarod. This is in agreement with behavioral clinical results stating that latency changes were of little consequence to the patient in terms of neurological function, preserving for example the visual acuity in MS [1].

In the case of crosstalk and leakage, up to a certain point demyelination made the phase between the front limbs increase and the amplitude of steps decrease, which resulted in a slower locomotion gait. We also observed a reversal of this tendency following higher demyelination damage, however this might be an artefact of our model due to the crosstalk framework, that will probably disappear when taking into account the axonal loss that occurs in very high demyelination percentages. The slowing of the locomotor gait means that for the task of walking on a rotarod turning at a constant speed, the mouse will have to increase the brainstem tonic input signal to maintain walking. However we also found that with higher tonic inputs that initiate faster walking gaits, demyelination caused more frequent non-walking transients by activating non-active limbs through crosstalk, stabilization into non-walking locomotor patterns with several limbs not touching the ground simultaneously or entered into a damped walking cycle that led to a simultaneous contraction of all the limb muscles. We conclude that the speed of the rotarod crucially affects the time the mouse can spend on it and needs to be a controlled variable of the behavioral experiments. Our model predicts that increasing the speed of the rotarod will significantly decrease the time the mouse can spend on it and this prediction could be easily verified by biological experiments.

Our model also predicts that, in locomotion, the amount of demyelination is less important than the location and distribution of the lesion. Highly dependent on inner feedback, the CPG model is mostly sensitive to temporal asymmetries in the onset of the input signal of different legs. The amplitude of the input signal is less critical since it governs the speed of the walking gait. When all the channels were impaired equivalently, the system settled in a walking gait, but asymmetries in the lesion (e.g., an axon bundle more affected than the others) were critical. We could show for example that nonwalking occurred with very low demyelination percentages with the same brainstem tonic input that always produced walking in the uniform lesion case. In addition, this would explain the counterintuitive results in [8] that show a weak correlation between demyelination and the time that the mouse could stay on the rotarod (i.e., in some cases behavior was worse with weaker demyelination). They concluded that factors other than lesion load, and mainly the axonal loss, contribute to neurological deficits. We believe that the distribution of the lesion is a critical factor and that this finding could possibly be explained by the fact that the more the spinal cord is demyelinated, the more the lesion would become symmetric leading to a lower behavioral deficit.

Finally the biological lesion had the most interesting and wide repertoire of behavioral deficits. It is difficult to directly relate the variety of non walking patterns we have found to precise biological observations, however they could provide an explanatory basis to the reduction of time that the demyelinated mice are able to walk on a rotarod [8]. Ideally we would like to have access to more biological results, such as for example patterns of EMG activity of muscles of the limbs, against which we would be able to validate our model, the data presented in [8] being rather sparse. Finally, we believe that a simple, but complete neural model of the demyelination function would possibly help us to gain more understanding in some of the deficits following demyelination and in a more broader sense MS, and to our knowledge this is the first neural model of that kind. In this paper for example, using a neural model of locomotion we could show that the actual distribution of the lesions and crosstalk are more damaging to behavior than the severity of lesions and delay respectively.

\section{REFERENCES}

[1] K. J. Smith and W. I. McDonnald. The pathophysiology of multiple sclerosis: the mechanisms underlying the production of symptoms and the natural history of the disease. Phil. Trans. R. Soc. Lond. B. vol. 354, pp. 1649-1673, 1999.

[2] S. Grillner, P. Wallen, K. Saitoh, A. Kozlov, and Robertson. B. Neural Bases of Goal-Directed Locomotion in Vertebrates - an Overview. Brain Res. Rev. (In Press), 2007. 
[3] J. F. Einum and J. T. Buchanan. Membrane Potential Oscillations in Reticulospinal and Spinobulbar Neurons During Locomotor Activity. $J$ Neurophysiol. vol. 94, pp. 273-281,, 2005.

[4] S. Grillner and P. Wallen. Cellular bases of a vertebrate locomotor system-steering, intersegmental and segmental co-ordination and sensory control. Brain Res. Rev. vol. 40, pp. 92-106, 2002.

[5] L. Zhaoping, A. Lewis and S. Scarpetta. Mathematical Analysis and Simulations of the Neural Circuit for Locomotion in Lampreys. Phys. Rev. Let., vol. 92, pp. 198106, 2004.

[6] A. J. Ijspeert. A connectionist central pattern generator for the aquatic and terrestrial gaits of a simulated salamander. Biol. Cybern., vol. 84(5), pp. 331-348, 2001.

[7] C. Pribe, S. Grossberg and M. A. Cohen. Neural control of interlimb oscillations. II. Biped and quadruped gaits and bifurcations. Biol. Cybern., vol. 77, pp. 141-152, 1997.

[8] D. B. McGavern, P. D. Murray, C. Rivera-Quinones, J. D. Schmelzer, P. A. Low and M. Rodriguez. Axonal loss results in spinal cord atrophy, electrophysiological abnormalities and neurological deficits following demyelination in a chronic inflammatory model of multiple sclerosis. Brain, vol. 123, pp. 519-531, 1997.

[9] N. Dale. Coordinated Motor Activity in Simulated Spinal Networks Emerges from Simple Biologically Plausible Rules of Connectivity. $J$ Comp. Neuro., vol. 14, pp. 55-70, 2003. 